\title{
Investigational New Drug
}

National Cancer Institute

\section{Source}

National Cancer Institute. Investigational New Drug. NCI Thesaurus. Code C49135.

A new drug or biological drug that is used in a clinical investigation. The term also includes

a biological product that is used in vitro for diagnostic purposes. 